\title{
Influences of elastane content, aesthetic finishes and fabric weight on mechanical and comfort properties of denim fabrics
}

\begin{abstract}
Denim has always been used for very durable outdoor work clothing because of its weight, rigidity and thickness. It is now more popular as comfortable and flexible clothing with stretch characteristics possessing elasticity so that it closely fits without restricting body movement. The aim of this paper is to investigate the effects of different aesthetic finishes applied while manufacturing, varied elastane content and fabric weight on its mechanical and stretch properties. It is observed that the tensile strength of denim fabric decreases with increasing elastane percentage, while breaking extension increases. Elastane content percentage shows significant influence on air permeability of denim. Air permeability of stretchable denim fabric decreases with increasing fabric weight. Flexural rigidity decreases with increasing elastane content and similar trend also for the drape coefficient. The increase in elastane content also influences the fabric stretch-ability with an increase in stretch percentage and decrease in fabric growth percentage with increasing elastane rate.
\end{abstract}

Keywords: air-permeability, drape-coefficient, elastane content, fabric weight, growth percentage, shrinkage
Volume 4 Issue I - 2018

\author{
Choudhary AK, Sheena Bansal \\ Department of Textile Technology, Dr B R Ambedkar National \\ Institute of Technology, India
}

\begin{abstract}
Correspondence: Dr.AK Choudhary, Department of Textile Technology, Dr BR Ambedkar National Institute of Technology, Jalandhar, Punjab, India PIN-1440II,Tel 9194I7710385,
\end{abstract} Email choudharyak@nitj.ac.in,akc1087@gmail.com

Received: June 15, 2017| Published: January 23, 2018

\section{Introduction}

The denim is a fabric woven from coarse indigo dyed warp and grey un-dyed weft yarn. Denim fabric is hard-wearing and has high density with a high mass per unit area and a 3/1 or 2/1-twill weaves construction. It is comfortable, fashionable, affordable, durable and a popular clothing material. Nowadays, the stretchable denims are in fashion due to its comfort fit and flexibility. These fabrics possess elasticity so that garments made out of stretchable denim fabric closely fit to body without restricting body movement. Clothes are mostly under strain in some parts of the body, such as the knee, elbow and lower back areas. Therefore, stretching is very important for the comfort of the wearer. Generally, fabrics are required to stretch comfortably in accordance with body movements, and also after stretching, to retain their original shape without any deformation. However, if clothes don't have such great flexibility, deformation occurs which is dubbed as fabric bagging. Özdil ${ }^{1}$ studied the stretch and bagging properties of denim fabrics containing different rates of elastane. The test results revealed that increasing the amount of elastane usage in denim fabric offers enhanced comfort properties. Mourad et al. ${ }^{2}$ studied the effects of elastane content percentage on physical and stretch properties of the plain woven cotton fabric. The results obtained in this study indicated that the amount of lycra has a significant influence on physical and elastic properties of woven fabrics. Fabric contraction increases with the increasing elastane content percentage. Fabric tensile strength decreases with Lycra rate, while fabric breaking extension increases because of the higher extension of Lycra fibers. Air permeability and tearing strength decreased significantly with the increase in lycra rate. This is because the fabrics will be thicker and more compact with the increase in lycra rate in the woven fabrics. It is observed that maximum stretch and fabric elastic recovery increases with Lycra rate fabric growth decreases. AL-ansary ${ }^{3}$ studied the effect of elastane content percentage on physical and mechanical properties of different woven fabrics including shrinkage $\%$, fabric growth and air- permeability and they revealed that the ratio of Lycra had a significant influence on the physical properties of woven fabrics.

There are two categories of stretchable denim fabrics, based on the degree of stretch-ability i.e. (a) power or action stretch and (b) comfort stretch. Power or action stretch provides a fabric with high degree of extensibility and quick recovery. The fabric stretch percentage generally ranges from at least $30 \%$ to $60 \%$ or more with no greater than $5 \%-6 \%$ recovery loss. Comfort stretch alludes to fabric with less than $30 \%$ stretch percentage and no greater than $2 \%$ to $5 \%$ recovery loss. ${ }^{4,5}$ Elastane yarns meet customer requirements of comfort, stretch-ability and appearance, so that again new trends are developed in denims using elastane yarns in the weft direction. Elastane yarns are more efficient in sports and comfort application. ${ }^{6}$ It gives sufficient elastic properties to the fabric even with $2 \%$ to $3 \%$ of elastane. Fabric handle refers to the total sensations experienced when a fabric is touched or manipulated in the fingers. ${ }^{7}$ The handle of the fabrics is affected by mainly fabric structure and fabric geometry. Fabric construction and yarn densities play major role in determining fabric handle. ${ }^{8,9}$ Fabric weight is related with warp and weft densities in the fabric. So, variations in warp and weft densities and in the number of warp and weft yarns have significant effects on the handle characteristics of the fabrics. ${ }^{8}$ The ratio of warp threads/cm to weft threads $/ \mathrm{cm}$ may result in increased yarn crimp, providing a potential increase in fabric extensibility in the warp direction. ${ }^{10}$ It was determined that the amount of elastane fibre in denim fabrics offers enhanced comfort properties. Therefore, as the elastane content increase, the fabric tensile and tearing strength decrease while stiffness increases. It is known that properties of fabric depend not only on its stated structural parameters, but also on formation conditions of fabric. It was determined that small differences of technological parameters do not influence fabrics without elastane, but have great influence on fabrics with elastane, because the elastic component of yarn is very sensitive for various effects Mourad et al. ${ }^{2}$ Fabrics with elastane especially are suitable for 
fitted clothes with large areas of stretch-ability. The influences of woven fabric stretch properties on garment design and pattern construction were evaluated. In order to analyze the elastic fabrics behavior during garment wearing the anisotropy of fabrics with elastane fiber due to their maximum strength and extension was investigated and new method was proposed. During wearer's movements, some parts of the garment experienced extension into opposite directions. As a result of such type extension, the complicated deformation consisting of shear, extension and buckling of material named as fabric structural mobility took place Özdil. ${ }^{1}$ Core spun yarns are used as a filling yarn in which core part is elastane filament to achieve desired stretch-ability in denim. The performance and comfort properties like stretching and recovery are the desirable characteristics of stretchable denim fabrics. In general the percentage of elastane varies from $1 \%$ to $5 \%{ }^{11,12}$ for optimum comfort performance of denim garments. Also fabric weight has significant effect on physical and mechanical properties like fabric thickness, fabric bending rigidity, tensile strength, and extension percentage and comfort parameters. So, it becomes necessary to know the combined effect of fabric weight and elastane percentage on drape, handle, durability, stretch performance, recovery and comfort factor of Denim garments, so that the garment manufacturers can design or construct the denim garments as per customer requirement. The objective of this experimental work is to explore the influences of type of finishes of manufacturing along with elastane content and fabric weight on physical and mechanical properties of denim.

\section{Experimental work}

\section{Materials}

In this experimental work cotton denim was used. Denim fabrics are provided with various kinds of finishes to enhance aesthetic surface appeal. Examples of various finishes applied to denim fabric are flat finish, fading through stone wash, acid wash or ozone, over dyeing or tinting, peach finish for velvet skin effect etc. As an overlook for all types of finishes three types of finishes were selected in this experimental as per below details:

Flat finished denim: It is a special process done to impart fabric with an even wash down effect and very clean surface. Originally liquid ammonia was used, but now uses mercerization plus calendaring processes to achieve the flat surface. Mercerization swells up the cotton fibers and allows the calendaring to press flat the surface.

Over dyed denim: This is an additional dyeing treatment. This adds another tone of color to the jeans. Normally denim garments is overdyed with yellowish dye for appearing dirty look. Tinted / over-dyed denim garments show a used/vintage $\&$ muddy look to the garments. There are various types of dyes used for over-dyeing/tinting purpose, such as:-Direct Dyes, Reactive Dyes, Pigment Dyes, and Sulphur Dyes. Tinting process done in the sample using sulphur dyes for 10 minutes followed by dye fixing \& clean-up of superficial dye.

Peach finished denim: This is a special finish mostly used in garments. Peach finish is given through light surface abrasion using fine brush or stones, which creates dense and short hairs layer on the fabric surface. These surface hairs will make fabric feel soft and bulkier.
Denim fabric samples are made from 12's Ne Yarns for both Warp and Weft with varied thread count to achieve required fabric weight levels of $250 \mathrm{~g} / \mathrm{m}^{2}$ and $300 \mathrm{~g} / \mathrm{m}^{2}$ in non-stretch denim fabric. For Stretch denim fabric, the yarn count for warp is 16's Ne and weft core spun yarn of $16 \mathrm{Ne}$ with elastane in the core. The elastane used in core of weft yarn was varied from 40 Denier to 55 Denier to achieve elastane content from $1.00 \%$ to $1.50 \%$. The thread count was varied to achieve fabric weight in stretch denim from $350 \mathrm{~g} / \mathrm{m}^{2}$ to $400 \mathrm{~g} / \mathrm{m}^{2}$, as given in Table 1.

Table I Experimental plan with various Finishes, Fabric Weight and Elastane Content percentage

\begin{tabular}{llll}
\hline $\begin{array}{l}\text { Fabric } \\
\text { Code }\end{array}$ & Finish & $\begin{array}{l}\text { Fabric Weight } \\
\left(\mathbf{g} / \mathbf{m}^{2}\right)\end{array}$ & $\begin{array}{l}\text { Elastane } \\
\text { Content }\end{array}$ \\
\hline $\mathrm{Cl}$ & Flat Finished & 250 & Nil \\
$\mathrm{C} 2$ & Denim & 300 & $\mathrm{Nil}$ \\
$\mathrm{O} 11$ & & 350 & $1.00 \%$ \\
$\mathrm{O} 12$ & & $1.50 \%$ \\
$\mathrm{O} 21$ & Over dyed Denim & & $1.00 \%$ \\
$\mathrm{O} 22$ & & 400 & $1.50 \%$ \\
$\mathrm{PI1}$ & & & $1.00 \%$ \\
$\mathrm{P} 12$ & & 350 & $1.50 \%$ \\
$\mathrm{P} 21$ & Peach Finish & & $1.00 \%$ \\
P22 & & 400 & $1.50 \%$ \\
\hline
\end{tabular}

\section{Testing parameters}

Breaking strength and breaking extension: Breaking strength of a yarn or fabric is defined as a maximum load that it will endure without breaking when subjected to uniaxial tensile loading. Breaking strength is the "load required to break the specimen". Breaking extension is the extension percentage at the breaking load. Breaking strength and extension percentage were tested on UTM at a speed of $300 \mathrm{~mm} / \mathrm{min}$. Fabric sample with $25 \mathrm{~mm} \times 150 \mathrm{~mm}$ gauge length as per ASTM standard D5035-11 was taken. Ten tests were carried out for each sample. Breaking Strength is expressed by the load at which the specimen breaks. The units used for breaking strength are Newton (N). Extension percentage is the strain expressed as a percentage rather than a fraction.

Drape-ability: Drape property of denim fabric is measured from the deformation by gravitational force of an initially horizontal fabric cut in the form of an annular ring. A circular fabric specimen is held concentrically between smaller horizontal disc and the lower supporting disc. Fabric is allowed to drape into graceful folds around the lower supporting disc. The outline of shadow is traced on paper and then its mass is determined. Initially, the weight of paper of $15 \mathrm{~cm}$ dia is determined and the drape co-efficient is calculated. Drape coefficient (F) is defined as the fraction of the area of the annular ring between the flat fabric edge and the supporting disc edge covered by the projection of the draped sample as shown in Figure 1. The Expression is given as equation (i).

$$
\text { Drape Coefficient, } F(\%)=\frac{(\text { Area under Drape Specimen }- \text { Area of disc })}{(\text { Area of Specimen }- \text { Area of Disc })} \times 100=\frac{\mathrm{W} 2-\mathrm{W} 3}{\mathrm{~W} 1-\mathrm{W} 3} \times 100
$$




\author{
Where \\ W1 - Area of Specimen, \\ W2 - Area under Drape Specimen \\ W3 - Area of disc
}

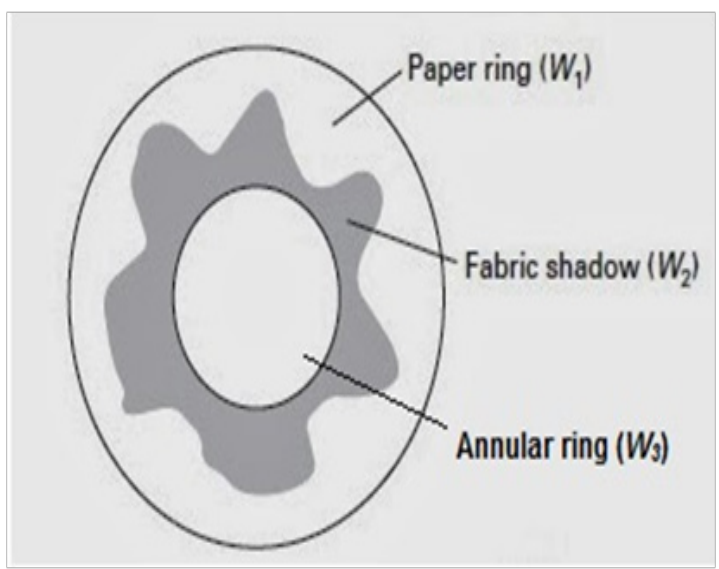

Figure I Fabric Drape diagram.

Flexural rigidity: The flexural rigidity is the measure of fabric stiffness. Denim fabrics are tested for flexural rigidity as per ASTM standard D1388-08 on the basis of principle is cantilever bending of the fabric under its own mass. The test was done on Cantilever Bending Tester. The sample of 8 inch $\times 1$ inch is cut from fabric. Move the clamped specimen by hand in a smooth manner at approximately $120 \mathrm{~mm} / \mathrm{min}$ until the edge of the specimen touches the knife edge as shown in Figure 2. Ten readings are carried out for each sample. Bending Length is calculated as per equation (ii). Higher the bending length, stiffer is the fabric. After that flexural rigidity is calculated using equation (iii).

Pierce empirical equation,

$$
C=L\left(\frac{\cos \theta / 2}{8 \tan \theta}\right)^{1 / 3}
$$

Where,

$$
\begin{aligned}
& \mathrm{M}=\text { Mass per unit area }\left(\mathrm{g} / \mathrm{m}^{2}\right) \\
& \mathrm{G}=\text { Flexural rigidity } \\
& \mathrm{C}=\text { Bending length }(\mathrm{mm}) \\
& \theta=\text { Angle fabric bends }
\end{aligned}
$$

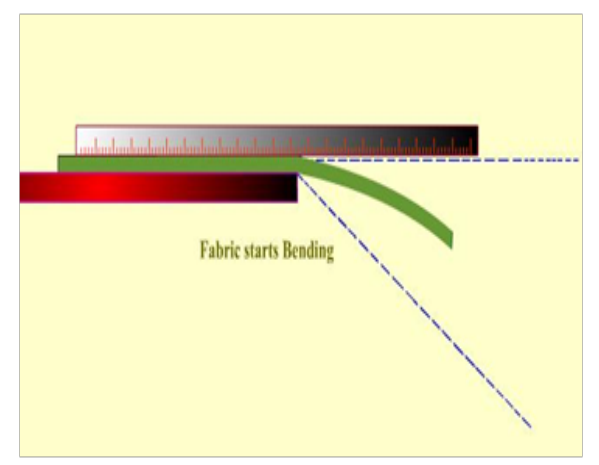

Figure 2 Cantilever Bending Tester.
Fabric flexural rigidity is calculated by following expression: Flexural rigidity,

$$
G=M \times C^{3} m g \cdot \mathrm{cm}^{3}
$$

Air permeability: Air permeability is an important factor in the performance of denim apparels. It depends on many factors such as; weave structure, fabric thickness, weight and porosity. From the previous studies it is determined that air permeability was affected significantly with the rate of spandex in weft yarns of the woven fabrics. As the rate of spandex in weft yarns increases, the air permeability decreases. Fabric air permeability tests were performed on machine model FX 3300 as per ASTM D737 standard. Total ten readings were taken from each fabric. Read and record the individual test results in SI units as $\mathrm{cm}^{3} / \mathrm{cm}^{2} / \mathrm{s}$

Stretchable properties: Fabric stretch-ability properties are related with fabric stretching percentage, fabric growth percentage and elastic recovery percentage. Fabric stretch-ability properties are tested as per ASTM standard D3107-07. The sample of $65 \mathrm{~mm} \times 560 \mathrm{~mm}$ was cut from fabric. Elastic performance is the ability of a material to exhibit its original properties after repeated use under cyclic loading and unloading, so fabric stretchable properties after cycling loading were also measured. Cyclic loading was done at three different target loads, i.e. $35 \%$ Breaking Load, $55 \%$ Breaking Load \& 75\% Breaking Load.

The total number of cycles required is ten, at fixed load. Sample strip length is same as used in stretchable testing of fabric.

And then calculated fabric stretching (eq. i), fabric growth (eq. ii) and elastic recovery (eq. iii) values at $35 \%, 55 \% \& 75 \%$ of Breaking Load:

Fabric stretching $(\%)=100 \times(\mathrm{B}-\mathrm{A}) / \mathrm{A}$

Fabric growth $(\%)=100 \times(\mathrm{C}-\mathrm{A}) / \mathrm{A}$

Elastic recovery $(\%)=100 \times(B-C) /(B-A)$

Where, A- The distance marked between the upper and bottom parts of the fabric $(250 \mathrm{~mm})$,

B- The distance between the marked points after 10 cycles of loading the sample (in ' $\mathrm{mm}$ ') and

C- The distance between the marked points after 1-hour relaxation.

\section{Results and discussions}

The test results for mechanical properties of Denim samples are given in Table 2.

\section{Influences on fabric mechanical properties}

In this study physical and mechanical properties include Breaking Strength (N) and Breaking Extension (\%):

Breaking strength: The breaking strength of all the fabrics in warp and weft directions is shown in Figure 3a. As per the graphical trend, the fabric weight and elastane content have significant effect on breaking strength of fabric. Breaking strength of peached finished denim fabric has significantly decreased in warp direction as compared to overdyed denim fabric due to surface fibre loss during peaching process. As fabric weight increases, the breaking strength increases in both warp and weft directions. The increase is less significant in case of non- stretch fabric warp direction. As elastane content increases, the 
breaking strength decreases for all fabric groups. Since elastane has lower tenacity than the cotton fiber, so increase in elastane content decreases breaking strength of fabric.

Breaking extension: The trend of breaking extension is represented in Figure 3b. Breaking extension of denim fabric is little higher for peached finished than over-dyed denim fabric due to increased surface fibre cohesion providing addition extension. Fabric weight increase results in increase of breaking extension in warp direction

Table 2 Mechanical properties and comfort parameters of fabric samples due to stiffer structure, while in weft direction the trend is completely opposite due to elastane in weft yarn. Breaking extension increases with increase in fabric weight in case of non-stretch fabric group due to inherent stretch of weft yarn crimp. Also for stretch fabrics, for first over-dyed finish group, the increase in breaking extension is more as compared to peach finished fabric group. As elastane content increases, the breaking extension increases in both directions, but the increase is much more significant in case of weft direction due to elastane present in weft yarn.

\begin{tabular}{|c|c|c|c|c|c|c|c|c|}
\hline \multirow[t]{2}{*}{ Fabric code } & \multicolumn{2}{|c|}{$\begin{array}{l}\text { Breaking } \\
\text { strength }(\mathbf{N})\end{array}$} & \multicolumn{2}{|c|}{$\begin{array}{l}\text { Breaking } \\
\text { extension (\%) }\end{array}$} & \multicolumn{2}{|c|}{$\begin{array}{l}\text { Flexural rigidity } \\
\left(\mathrm{mg} / \mathrm{cm}^{3}\right)\end{array}$} & \multirow[t]{2}{*}{$\begin{array}{l}\text { Drape } \\
\text { coefficient }\end{array}$} & \multirow[t]{2}{*}{$\begin{array}{l}\text { Air permeability } \\
\left(\mathrm{cm}^{3} / \mathrm{s} / \mathrm{cm}^{2}\right)\end{array}$} \\
\hline & Warp & Weft & Warp & Weft & Warp & Weft & & \\
\hline $\mathrm{Cl}$ - No Elastane & 528 & 195.4 & 29.07 & 27 & 276.97 & 67.18 & 0.608 & 22.65 \\
\hline C2 - No Elastane & 529 & 247 & 29.33 & 30.87 & 386.81 & 186.04 & 0.682 & 24.47 \\
\hline OII - $1.00 \%$ Elastane & 922 & 264.6 & 37.57 & 35.5 & 1718.29 & 297.08 & 0.81 & 10.36 \\
\hline OI2 - $1.50 \%$ Elastane & 756 & 250 & 34.8 & 54.2 & $180 \mid .7$ & 215.6 & 0.78 & 4.45 \\
\hline $\mathrm{O} 21-1.00 \%$ Elastane & 1055.5 & 308.8 & 40.1 & 28.06 & 1981.5 & 340.12 & 0.88 & 9.65 \\
\hline O22 - $1.50 \%$ Elastane & 865 & 290.5 & 37.07 & 48.37 & 2059.12 & 222.23 & 0.83 & 3.65 \\
\hline PII - I.00\% Elastane & 652 & 287.4 & 42.9 & 82.6 & 1903.54 & 757.4 & 0.78 & 4.923 \\
\hline $\mathrm{PI} 2$ - I.50\% Elastane & 632 & 260.5 & 42.33 & 84.5 & 2091.53 & 645.59 & 0.61 & 2.401 \\
\hline P2I - I.00\% Elastane & 746 & 327.8 & 46.9 & 72.8 & 2111.78 & 845.99 & 0.87 & 3.863 \\
\hline P22 - I.50\% Elastane & 725 & 324.5 & 48.37 & 79.18 & 2387.29 & 675.32 & 0.75 & 2.102 \\
\hline
\end{tabular}

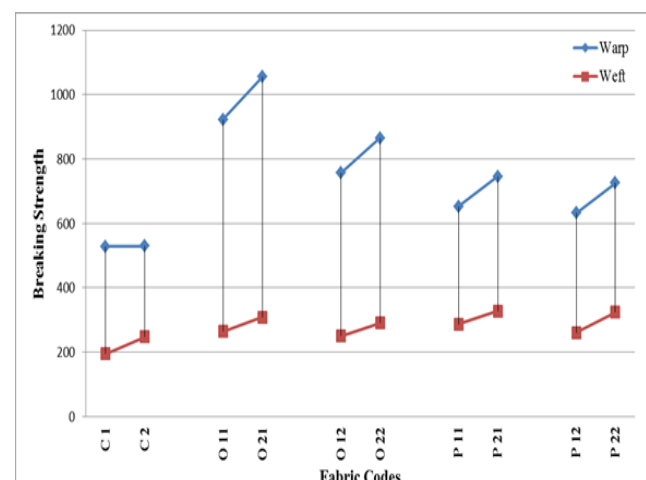

Figure 3a Influence of fabric weight and elastane content on breaking strength in warp and weft direction.

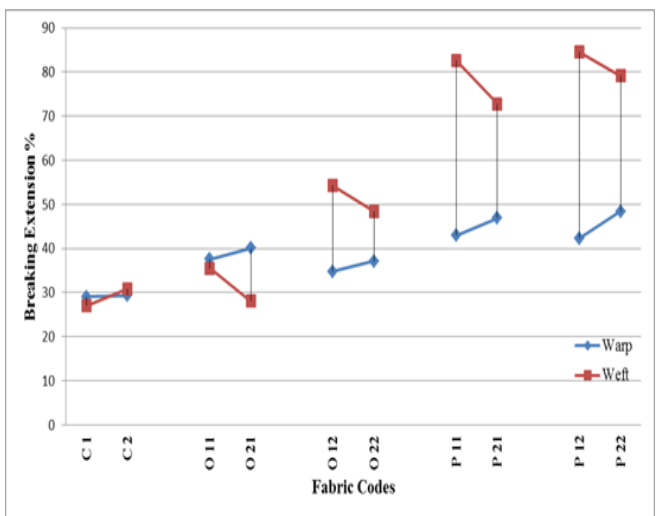

Figure 3b Influence of fabric weight and elastane content on breaking extension percentage in warp and weft direction.
Fabric comfort parameters include air permeability, flexural rigidity and drape coefficient of the fabric.

Air-permeability: The trend of air permeability of sample group is showed in Figure 4. Peach-finished denim fabric air permeability is lesser due to lose fibrous layer present on surface restricting air flow from the fabric. Also air permeability is the highest in case of nonstretch fabric as elastane makes the fabric structure more compact. As fabric weight increases, the air permeability decreases due to more tight structure, while air permeability increases in case of nonstretch fabric due to open structure and coarser yarn count usage, the fabric cover has been decreased. As elastane content increases, the air permeability decreases for all fabric groups.

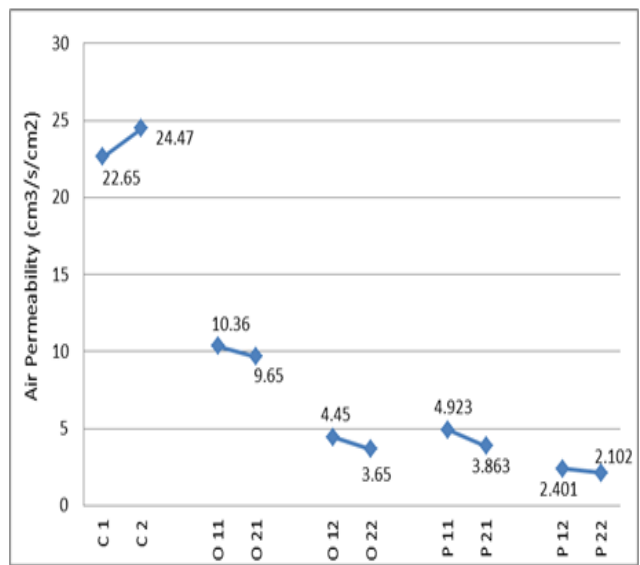

Figure 4 Influence of fabric weight and elastane content on air permeability. 
Flexural rigidity: Flexural Rigidity as shown in Figure 5 is more in warp direction due to more warp thread count than weft thread count. Peached Finished Denim Fabric shows higher flexural rigidity as compared to over-dyed and very high as compared to flat finished non-stretch fabric. This is because the more the processing, more the fabric becomes stiff. As fabric weight increases, the flexural rigidity increases due to more tight structure. As elastane content increases, flexural rigidity increases in warp direction while decreases in weft direction.

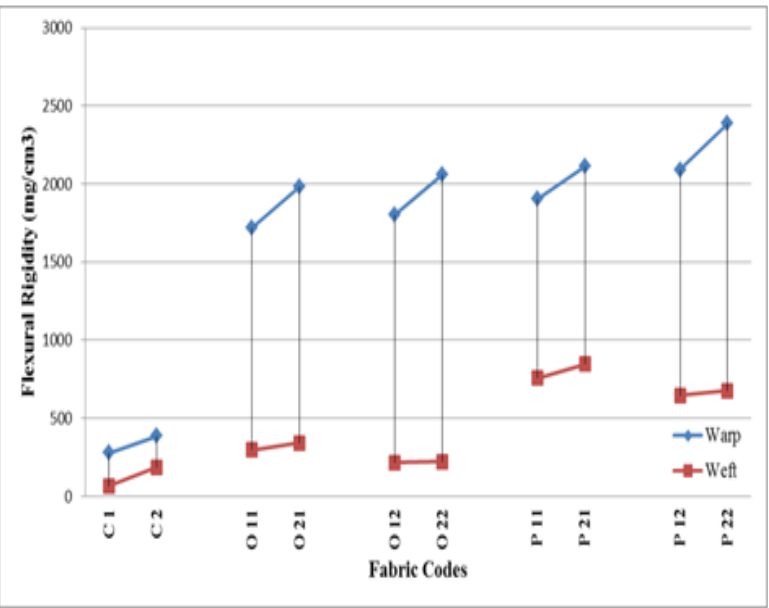

Figure $\mathbf{5}$ Influence of fabric weight and elastane content on flexural rigidity.

Drape-coefficient: Drape coefficient has a similar trend as flexural rigidity in context of change in fabric weight, as shown in Figure 6a. Also the trend due to effect of elastane content variation shown in Figure $6 \mathrm{~b}$. As elastane content increases, drape coefficient of denim fabric decreases; which indicates that fabric stiffness decreases with increased content of elastane.

\section{Influences on fabric stretch-ability properties}

As mentioned earlier, the effect on stretchable properties is analyzed under cyclic loading of ten cycles each at three levels of Percentage of Breaking Load of fabric. Fabric Stretch(\%), Fabric Growth(\%) and Elastic Recovery\% were calculated on above data. Test results for stretch-ability properties (Fabric stretch and growth percentage, elastic recovery percentage) are given in Table 3.

Fabric stretch properties: Graphical representation of Stretch properties is shown in given Figures 7 a. Stretch percentage, b. Growth percentage and c. Elastic recovery percentage.

Peached Finished Denim Fabric Stretch percentage is higher at all the three breaking load due to fibre cohesion on surface. As fabric weight increases, the stretch percentage decreases due to tighter and stiffer structure. As elastane content increases, the stretch percentage increases for all fabric groups. Since elastane makes the structure more compact. Peached Finished Denim Fabric growth percentage is higher at all the three breaking load due to fibre cohesion on surface. As elastane content increases, the growth percentage decreases for all fabric groups. Peached Finished Denim Fabric elastic recovery percentage is lower as compared to over-dyed fabric but higher than flat finished at all the three breaking load. Peaching process abrades the top most sheath layer of weft core spun yarn, due to which lycra in core can stretch more easily, thus stretch percentage and fabric growth is more than other two sample groups, while stretch recovery is less after repeated stretching because the recovery due to sheath fibres is lesser as compared to other two cases. Flat finished fabric elastic recovery is less as compared to over dyed and peach finished denim, as this is just the inherent stretch of fabric due to weft crimp. So, there is no elastane which can help the structure to recover back. As fabric weight increases, the elastic recovery percentage increases due to tighter and stiffer structure. As elastane content increases, the elastic recovery percentage increases for all fabric groups. Since elastane makes the structure more stable.

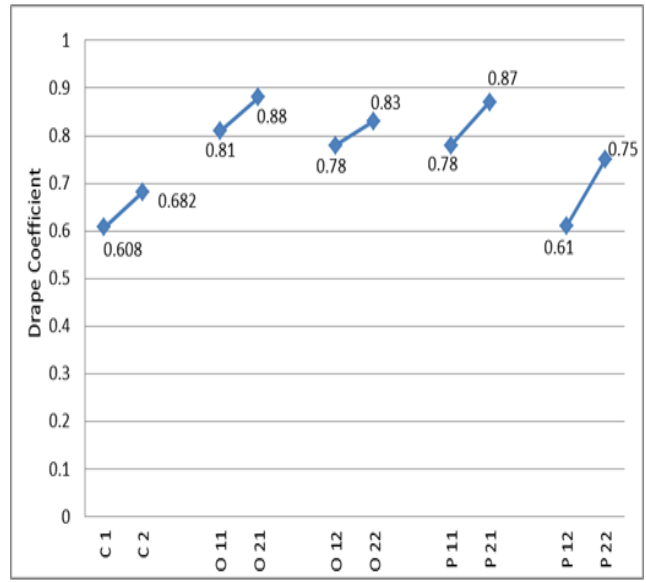

Figure $\mathbf{6 a}$ Influence of fabric weight on drape-coefficient.

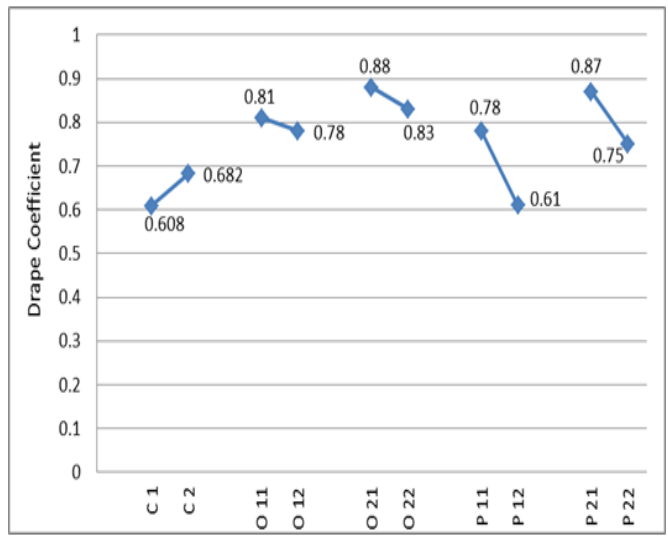

Figure $\mathbf{6 b}$ Influence of elastane content on drape-coefficient.

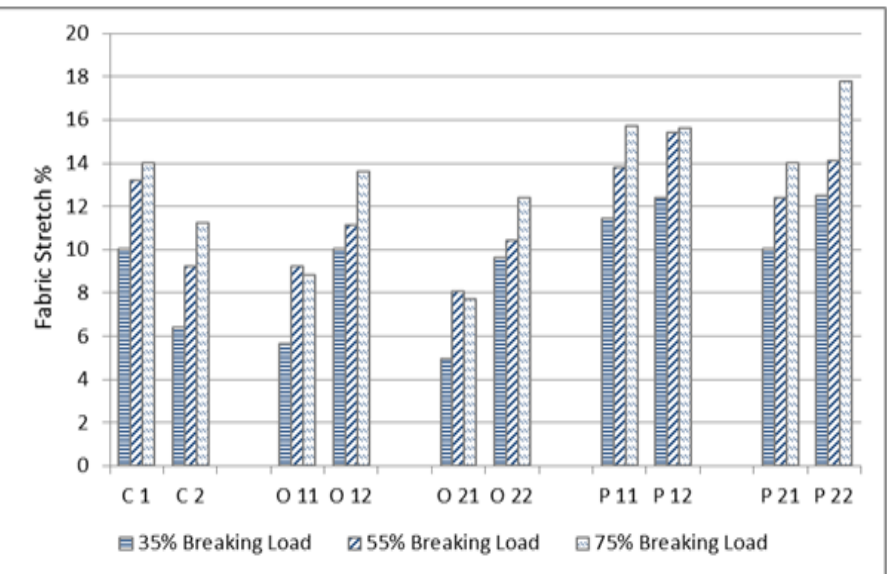

Figure 7a Effect of fabric weight and elastane content on fabric stretch percentage. 


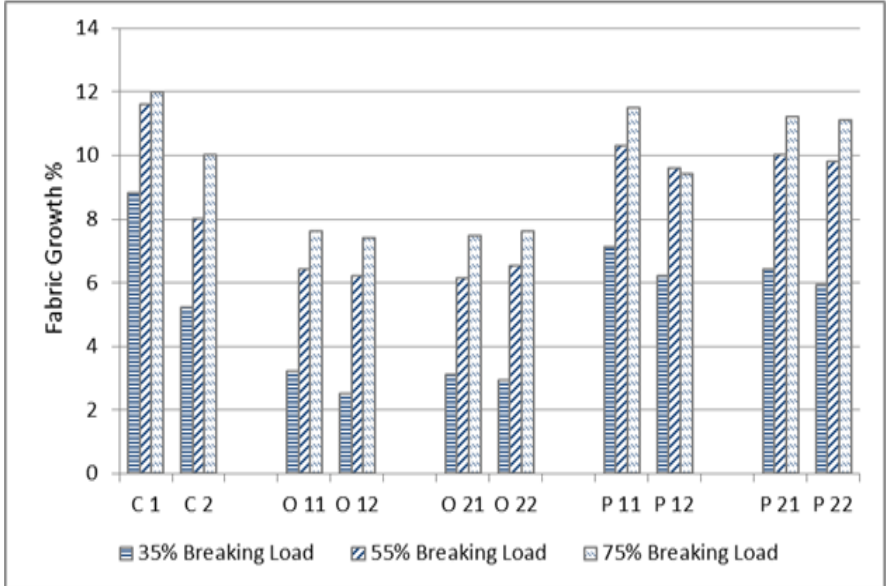

Figure 7b Effect of fabric weight and elastane content on fabric growth percentage.

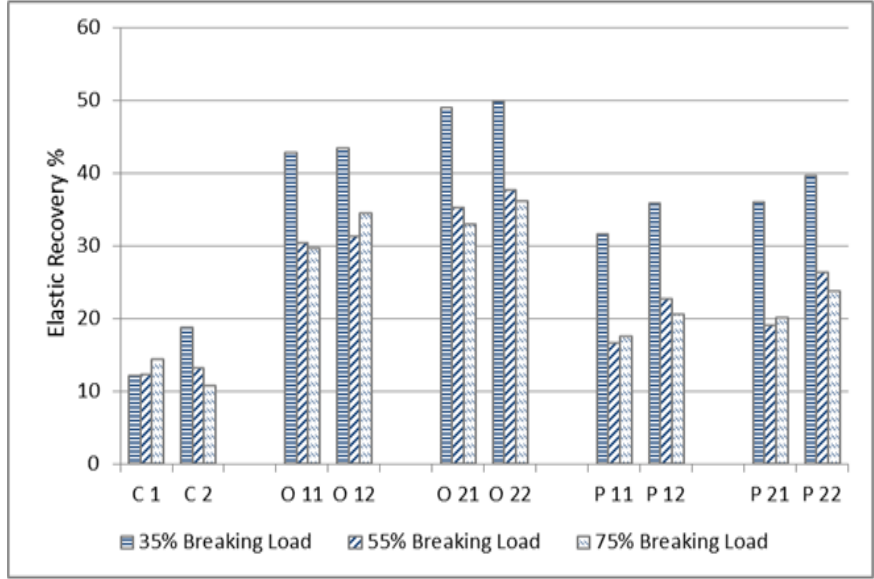

Figure 7c Effect of fabric weight and elastane content on fabric elastic recovery percentage.

Table 3 Test Results for Stretch-ability Properties

\begin{tabular}{|c|c|c|c|c|c|c|c|c|c|}
\hline \multirow[t]{3}{*}{ Fabric code } & \multicolumn{9}{|c|}{$\begin{array}{l}\text { Breaking load } \\
(\mathrm{N})\end{array}$} \\
\hline & $35 \%$ & $55 \%$ & $75 \%$ & $35 \%$ & $55 \%$ & $75 \%$ & $35 \%$ & $55 \%$ & $75 \%$ \\
\hline & \multicolumn{3}{|c|}{ Fabric stretch (\%) } & \multicolumn{3}{|c|}{ Fabric growth (\%) } & \multicolumn{3}{|c|}{ Elastic recovery (\%) } \\
\hline $\mathrm{Cl}$ - No Stretch & 10 & 13.2 & 14 & 8.8 & 11.6 & 12 & 12 & 12.12 & 14.28 \\
\hline C2 - No Stretch & 6.4 & 9.2 & 11.2 & 5.2 & 8 & 10 & 18.75 & 13.04 & 10.71 \\
\hline OII - I.00\% Elastane & 5.6 & 9.2 & 8.8 & 3.2 & 6.4 & 7.6 & 42.8 & 30.4 & 29.6 \\
\hline OI2 - I.50\% Elastane & 10 & 11.1 & 13.6 & 2.5 & 6.2 & 7.4 & 43.3 & 31.3 & 34.4 \\
\hline $\mathrm{O} 2 \mathrm{I}-1.00 \%$ Elastane & 4.9 & 8.05 & 7.7 & 3.07 & 6.12 & 7.46 & 48.9 & 35.2 & 32.9 \\
\hline O22 - I.50\% Elastane & 9.6 & 10.4 & 12.4 & 2.9 & 6.5 & 7.6 & 49.83 & 37.61 & 36.03 \\
\hline PII - I.00\% Elastane & 11.42 & 13.8 & 15.7 & 7.1 & 10.3 & 11.5 & 31.5 & 16.6 & 17.5 \\
\hline $\mathrm{PI} 2$ - I.50\% Elastane & 12.4 & 15.4 & 15.6 & 6.2 & 9.6 & 9.4 & 35.76 & 22.58 & 20.5 \\
\hline P2I - I.00\% Elastane & 10 & 12.4 & 14 & 6.4 & 10 & 11.2 & 36 & 19 & 20 \\
\hline P22 - I.50\% Elastane & 12.5 & 14.1 & 17.8 & 5.9 & 9.8 & 11.1 & 39.6 & 26.2 & 23.6 \\
\hline
\end{tabular}

\section{Conclusion}

The results obtained in the study clearly indicate that the type of finish, fabric weight and amount of elastane have a significant influence on physical and elastic properties of denim fabrics. It is observed that breaking strength, flexural rigidity, fabric stretch percentage and fabric growth percentage in stretch fabrics are higher in peach finished denim fabric. Breaking extension, air permeability, drape coefficient and fabric elastic recovery percentage reduce due to fibre loss and structural changes during peaching. Fabric weight also influences the physical and elastic properties of denim fabrics for both- stretch and non- stretch fabrics. Flexural rigidity, breaking strength, breaking extension percentage and drape coefficient increases with increase in fabric weight. At a fixed level of elastane content the fabric stretch decreases as fabric weight increases, owing to the more inter yarn friction; whereas the fabric growth increases due to the less recovery of the fabric. Air permeability of stretchable denim fabric decreases with increase in fabric weight. Fabric structure becomes compact with the increase in elastane content. Fabric tensile strength decreases with spandex rate, while fabric breaking extension increases because of the higher extension of spandex fibers. Air permeability and tensile

strength decreased significantly with the increase in spandex rate. This is because the fabrics become thicker and more compact with the increase in spandex rate in the woven fabrics. Above graphical trends proved that maximum stretch and fabric elastic recovery increase with spandex percentage inside fabric. On the other hand, there is inverse relationship between spandex percentage and fabric growth. Elastane content has a significant influence on air permeability as the fabric structure becomes compact. The structure compactness will be higher with the increase in the elastane content, which in turn leads to the woven fabrics becoming more thicker, and then less permeable to air flow.

\section{Acknowledgements}

This is original work done by us in our departmental lab (NIT Jalandhar). We also certify that this work is not communicated to any other Journal for publication.

\section{Conflict of interest}

There is no conflict of interest. 


\section{References}

1. Özdil N. Stretch and bagging properties of denim fabrics containing different rates of elastane. Fibres \& Textiles in Eastern Europe. 2008;1(66):63-67.

2. Mourad MM, Elshakankery MH, Almetwally AA. Physical and stretch properties of woven cotton fabrics containing different rates of spandex. J American Science. 2012;8(4):567-572.

3. AL-Ansary MAR. Effect of spandex ratio on the properties of woven fabrics made of cotton/spandex spun yarns. J American Science. 2011;7(12):63-67.

4. Haque MM. Effect of weft parameters on weaving performance and fabric properties. J Science \& Technology. 2009;4(2):62-68.

5. Sinha SK, Bansal P, Maity S. Tensile and elastic performance of cotton/ lycra core spun denim yarn. J Institution Engineers. 2017;89(1):71-78.

6. Bardhan MK, Sule AD. Anatomy of sportswear and leisurewear: Scope for spandex fibres. Man Made Text India. 2001;44:81-86.
7. Das A, Ishtiaque SM. Comfort characteristics of fabrics containing twist-less and hollow fibrous assemblies in weft. $J$ Textile \& Apparel. 2004;3(4):1-8.

8. Shanmugasundaram OL. Objective measurement techniques for fabrics. Asian Textile J. 2008;17:63-67.

9. Na Y, Kim C. Quantifying the handle and sensibility of woven silk fabrics. Textile Research J. 2001;71(8):739-742.

10. Nassif GAA. Effect of weave structure and weft density on the physical and mechanical properties of micro polyester woven Fabrics. J American Science. 2012;8(8):947-952.

11. Kumar MS, Anbumani N, Hayavadana J. Elastane fabric - A tool for stretch applications in sports. Indian J Fibre \& Textile Research. 2011;36(3):300-307.

12. Ortlek HG, Ulku S. Effects of spandex and yarn counts on the properties of elastic core-spun yarns produced on murata vortex spinner. Text Res J. 2007;77(6):432-436. 